\title{
Use of Virtual Reality Glasses to Reduce Child Pain and Fear during Painful Medical Procedures in Children with Physical Disabilities in Uganda: A feasibility study
}

\author{
Femke Bannink Mbazzi 1,2,3, Claire Nimusiima ${ }^{1}$, Daniella Akellot ${ }^{4}$, Elizabeth Kawesa ${ }^{1,4}$, Sarah Hodges ${ }^{4}$, Janet Seeley ${ }^{1,3}$, Tine Vervoort ${ }^{2}$ \\ ${ }^{1}$ MRC/UVRI \& LSHTM Uganda Research Unit, Entebbe, Uganda; ${ }^{2}$ Faculty of Psychology and Educational Sciences, Ghent University, Ghent, Belgium; ${ }^{3}$ London School of \\ Hygiene \& Tropical Medicine, Global Health \& Development, London, United Kingdom; ${ }^{4}$ CoRSU Rehabilitation Hospital, Kisubi, Uganda \\ Funded by: Wellcome's Institutional Strategic Support Fund, LSHTM, 204928/Z/16/Z \& Post doc fellowship 12V7120N Research Foundation Flanders (FWO).
} Corresponding author: femke.bannink@ugent.be / femke.bannink@lshtm.ac.uk

\section{BACKGROUND}

The use of distraction to effectively reduce pain and fear in children during painful medical procedures has been widely documented in high income countries. This study explored the acceptability and feasibility of the use of lowcost virtual reality (VR) glasses, and the WongBaker Faces Pain Scale and Children's Fear Scale scales, for pain and fear reduction in children admitted at CoRSU Rehabilitation Hospital in Uganda.

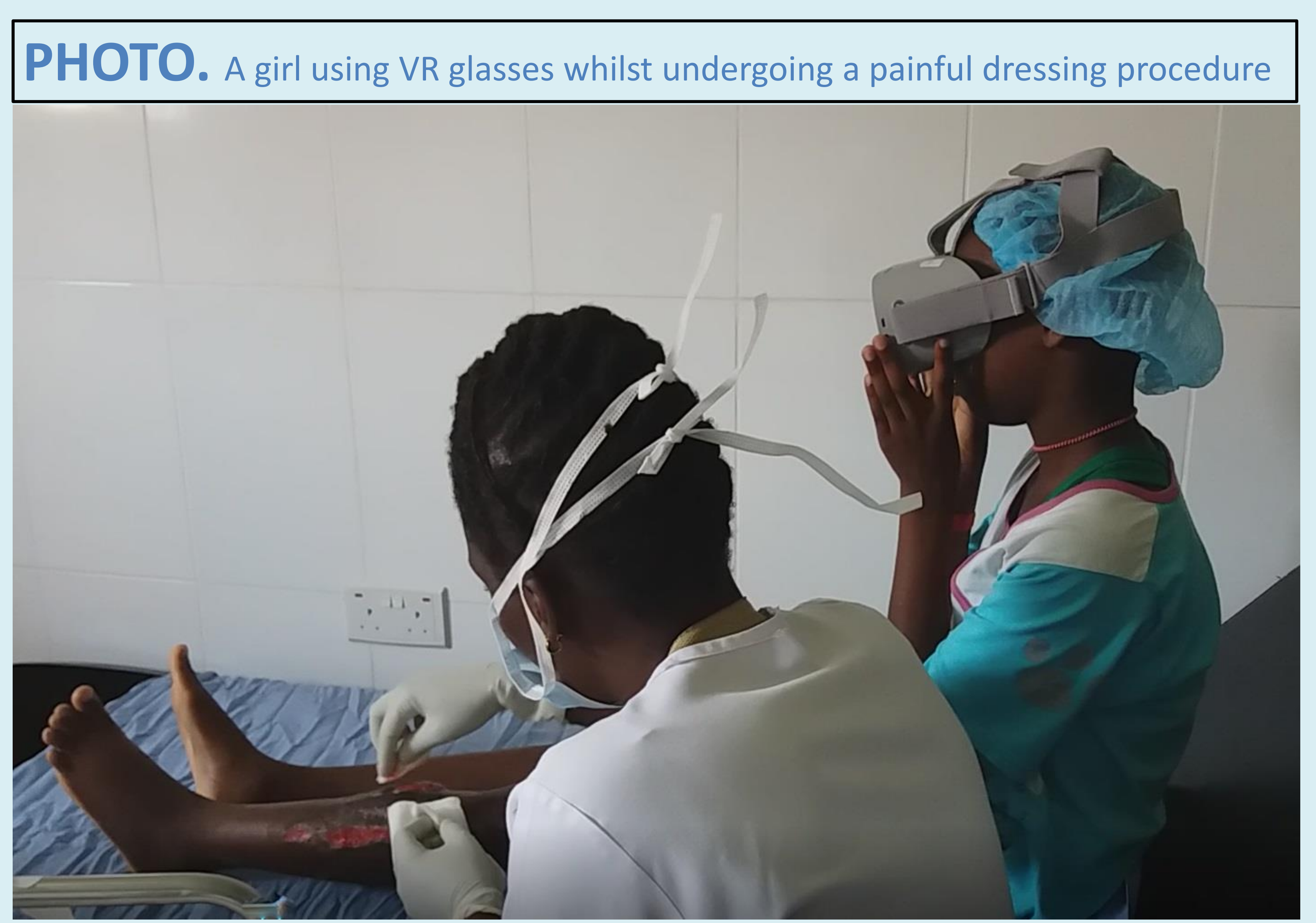

\section{METHODS}

- All children undergoing painful dressing procedures, were offered to wear a Oculus Go Standalone VR Headsets and watch 3D children's cartoons during the procedure.

- Study staff registered if the child declined, hesitated but accepted after encouragement, or accepted to use the glasses at once.

- Children were requested to indicate the pain they were experiencing using the Wong-Baker Faces Pain Scale (FACES) as a pain measurement tool, and fear they had about the procedure using the Children's Fear Scale (CFS) as a fear measurement tool before and after the dressing procedure.

- Caregivers of the children were asked about their own procedure-related fear before and after the procedure too.

- As these pain and fear assessment tools have not been used extensively amongst comparable African populations, children were also asked to what extent they felt faces depicted in the FACES and CFS represented pain and fear expressions, respectively.

- Acceptability and feasibility was further explored in 5 focus group discussions (13 children, 10 caregivers and 9 nurses).

- Quantitative data were analysed using STATA15. Qualitative data were transcribed and analysed in NVIVO12.

\section{STUDY PARTICIPANTS}

Participants consisted of 79 children (32\% female, 68\% male), with a median age of 13 (range 4-17 years, SD=3.13) who were treated for osteomyelitis (77\%), pressure sores (5\%), and other conditions (17\%), hospitalized at CoRSU Hospital. The procedures included general wound dressing (35.4\%), and wound dressing post-sequestrectomy (74.9\%). Less than $4 \%$ of the children who participated were offered pethidine IM (2.5\%), a ketamine mixture containing paracetamol syrup, diazepam and ketamine $(0.9 \%)$, paracetamol $(0.3 \%)$, or diclofenac $(0.3 \%)$ in addition to the VR glasses. The average length of the procedure was 21 minutes ( $S D=15$, range $2-129$ minutes).

\section{RESULTS}

- $85.9 \%$ (79/92) caregivers agreed to participate in the study. The caregivers who declined to participate expressed concerns that this new device might cause cancer or infertility.

- $96 \%$ of the participating children immediately accepted and used the VR glasses. $2.53 \%$ hesitated but later accepted the use of the glasses, and $1.27 \%$ declined to use the glasses.

- $19.8 \%$ of the children asked to remove the glasses during the procedure. The request to remove the glasses during the procedure reduced to $7.4 \%$ in subsequent exposures. On average each child was exposed to using the glasses 5.15 times $(S D=4.39$, range $1-21)$ during their admission.

'It takes away all my fears' (15 year old male participant)

'The pain is less compared to previous dressings, I am happy to watch' (11 year old female participant)

'The glasses make her [the child] not focus so much on the dressing, they reduce her fear and keep her calm' (mother to an 8 year old female participant)
- Children, caregivers, and nurses found the glasses helpful in distracting children from the medical procedure, and felt the use of the glasses helped reduce child fear and pain.

- Nurses felt it made their work less stressful as children tended to cry less and be more calm during the procedure.

- The FACES was an acceptable and feasible method to measure pain, whilst our study population felt the faces on the CFC were hard to read and identify with.

\section{CONCLUSION}

Acceptability of VR glasses in children in our study overall was high; positive reactions were gathered from children, parents, and nurses. The use of VR glasses may offer an acceptable and effective pain and fear reduction method in resource-constrained settings and should be further explored in a randomized control trial. 